\title{
The Effect of Blood Velocity Change after SUPER LIZER on Rotator Cuff Pain
}

\author{
Oкchul Hwang, PhD ${ }^{1)}$, Kyungun Ha, MS, PT $^{2)}$ \\ 1) College of Arts, School of Sports and Health \\ 2) Department of Physical Therapy, Dong-A University Medical Center: 3-g, Dongdaesin-Dong, \\ Seo-Gu, Busan, 602-715, Republic of Korea. TEL: +82 51-240-5650, FAX: +82 51-248-1510, E-mail: \\ Ha8311@gmail.com
}

\begin{abstract}
Purpose] We hypothesized that rotator cuff patients would see dramatic pain reduction after linear polarized near infrared irradiation of the rotator cuff muscles. [Subjects] The subjects of this study were 30 rotator cuff injury patients who visited a hospital's rehabilitation treatment room from September to December 2011. Irradiation was administered three times per week by a single operator in order to minimize variables affecting this study. [Methods] The subjects were randomly allocated to either an experimental group (range of motion exercise and then irradiation) and a control group (range of motion exercise only). [Results] The numbers of males and females were 19 and 21, respectively. The average age of the experimental group was $46 \pm 4.34$ years old and that of the control group was $46.93 \pm 6.17$. There were no statistical differences in the demographic characteristics of the two groups. The experimental group's pain was found to be significantly less severe than that of the control group after the intervention. The experimental group's blood flow velocity was faster than that of the control group, but the difference was not statistically significant. [Conclusion] This study showed that Super Lizer irradiation was effective at reducing rotator cuff pain. The irradiation also increased blood flow volume. However, a blood flow volume increase was also observed when joint exercise was performed, which suggests that blood flow volume increase was not a treatment effect of the irradiation. Super Lizer appears to be effective at reducing the pain of rotator cuff patients.
\end{abstract}

Key words: Rotator cuff patients, Blood flow velocity, Super Lizer

(This article was submitted Apr. 3, 2012, and was accepted Apr. 26, 2012)

\section{INTRODUCTION}

Senile diseases are diversifying and the number of elderly patients is growing as people's average life expectancy increases. In particular, pain from shoulder joint diseases resulting from degenerative change in the musculoskeletal system, ranks second to lumbar pain in its frequency, with a prevalence of 6.6 to 25 out of a population of $1,000^{8)}$. Diseases that cause shoulder joint pain are various. It is most important to identify causative diseases of shoulder joint pain, as in pain on other areas of the body. Rotator cuff syndrome is a common shoulder joint disorder, and in many cases patients with this syndrome face difficulty with treatment largely due to exercise or administration of agents without a precise diagnosis.

Rotator cuff syndrome is the most common disorder among intra-articular diseases causing pain, which include rotator cuff rupture and impingement syndrome ${ }^{6}$. Rotator cuff damage is known to result from different factors such as intrinsic degeneration of the area itself or inflammatory response, to subacromial impingement due to internal causes.

In a sample study of partial rotator cuff rupture, Fukuda et al. ${ }^{5)}$ asserted that an inflammatory response, induced by subacromial mucous cysts, causes the condition in patients. Growing attention is being paid to treatment methods such as pain alleviation through blood flow improvement within the muscles by increasing hand temperature.

Super Lizer (HA-550S, Iken, Japan) produces linear polarized near infrared radiation. Near infrared radiation, as a treatment for blood flow improvement or blood flow volume increase of the rotator cuff muscles controlled by the cervical spinal nerves, has been found to bring about significant blood flow improvement around the affected region ${ }^{1)}$.

We hypothesized that rotator cuff patients would see dramatic pain reduction after linear polarized near infrared irradiation treatment of the rotator cuff muscles.

\section{SUBJECTS AND METHODS}

The subjects of this study were 30 rotator cuff injury patients who visited the rehabilitation treatment room of $\mathrm{X}$ Hospital from September to December 2011. Irradiation was administered three times per week by a single operator in order to minimize variables affecting this study.

The subjects were randomly allocated to either the experimental group (range of motion exercise and then irradiation) or the control group (range of motion exercise only). Blood 
flow volume in the rotator cuff of the affected side was measured using an ultrasonic blood flow meter (ES-1000SP II, Hedeco, Japan). The intensity of pain was estimated using a visual analogue scale (VAS).

The patients gave their prior consent to participation in the experiment.

Blood flow velocity and pain of the experimental group and the control group were compared before and after Super Lizer irradiation using group averages. The linear mixed model was employed in order to verify whether there were differences between the two groups and within each group before and after the intervention. Further, a multiple comparison was made using Tukey's studentized range test. A significance level of 5\% was used for all analyses. The SAS 9.2 program was used for statistical analysis.

\section{RESULTS}

The numbers of males and females in the subjects were 19 and 21, respectively. The age of the experimental group was $46 \pm 4.34$ years old, and they had an average body weight of $67.31 \pm 5.85 \mathrm{~kg}$, and an average height of $162.34 \pm 5.56 \mathrm{~cm}$. The average age of the control group was $46.93 \pm 6.17$ years old, and they had an average body weight of $68.21 \pm 3.54 \mathrm{~kg}$, and an average height of $166.28 \pm 7.48 \mathrm{~cm}$. There were no statistical differences in demographic or anthropometric characteristics between the two groups.

Using the linear mixed model, we examined statistical differences in the degree of pain between the experimental group and the control group at the pre- and post-test time points. Reciprocal actions between the treatment groups and their treatment time points, effects between the treatment groups, and effects between the treatment time points were statistically significant $(\mathrm{p}<0.001)$. The above result indicates that post-test changes in the degree of pain were significantly different in the experimental and control groups, as clearly shown by their average profile graph (Fig. 1). The experimental and control groups' degree of pain had decreased from at the post-test time point.

For more detailed verification, we conducted the TukeyKramer multiple comparison test (Table 1). The differences in the average values (with a 95\% confidence interval) of the degree of pain between the pre- and post-test time points were statistically significant $(p<0.001)$ in both the experimental and control groups. At the pre-test time point, the difference in the average pain values between the experimental group and the control group was not statistically significant $(\mathrm{p}=0.500)$; however, the difference in the average pain value at the post-test time point was statistically significant $(\mathrm{p}<0.001)$.

Overall, both groups were homogeneous in their degree of pain at the pre-test time point and both groups showed statistically significant decreases in degree of pain at the post-test time point. Furthermore the experimental group's degree of pain had decreased significantly more than that of the control group, using the linear mixed model, we also looked at differences in blood flow velocity of the experimental and control groups. Differences between the test time points were statistically significant $(\mathrm{p}<0.001)$. However, reciprocal action between the treatment groups and their test time points and differences between the treatment groups were not statistically significant. This means that changes in blood flow velocity between the treatment groups were not significantly different, as clearly shown by the average profile graph (Fig. 2). The experimental and control groups' blood flow velocity had decreased at the post-test time point.

For more detailed verification, we performed the TukeyKramer multiple comparison test (Table 2). The differences in the average values (with a $95 \%$ confidence interval) of blood flow velocity between the pre- and post-test time statistically significant $(p<0.001)$ in both groups. The differences in the averages value of blood flow velocity between the experimental group and the control group at the pre and post-test time points were not statistically significant $(\mathrm{p}=0.500$, and $\mathrm{p}=0.690$, respectively).

Overall, both groups were homogeneous in their blood flow velocity at the pre-test time point and showed statistically significant decreases in blood flow velocity at the post-test time point. Changes in blood flow velocity did not show any significant difference between the groups, almost the same degree of change.

\section{DISCUSSION}

We that Super Lizer treatment would be effective at alleviating rotator cuff pain, since its known effects are blood flow improvement and repair of damaged muscle tissues ${ }^{2}$.

High power lasers are used as laser scalpels for surgical operations, and low power lasers, He-Ne lasers or semiconductor lasers, are used selectively to treat skin lesions or rheumatism, locally. Super Lizer, a kind of low power laser, is a new light treatment system. It has potential in breaking the vicious circle of pain by relaxing contracted peripheral blood vessels caused by excessive tension of the sympathetic nervous system, prompting blood circulation, and aiding excretion of algogenic substances such as bradykinin ${ }^{4,7,9)}$.

Bonelli et al. ${ }^{3}$ ) observed that increased blood flow volume in the skin alleviated pain. Our present study attempted to reduce pain by increasing blood flow velocity in the rotator cuff. Super Lizer treatment resulted in increased blood flow volume in the skin and statistically significant pain alleviation.

When initiating this study, we considered the fact that patients' complaints of pain were very subjective and pain intensity greatly varied among them. The subjects of this present study were randomly allocated to either the experimental group (range of motion exercise and irradiation) or the control group (range of motion exercise only). Super Lizer intervention was repeatedly applied. The outcome was that the experimental group, which received a combined therapy of joint exercise and Super Lizer irradiation, reported a large decrease in their subjective pain scores, but blood flow volume change was not correlated with the pain alleviation.

This study showed that Super Lizer irradiation was effective at reducing rotator cuff pain. Super Lizer irradiation also increased blood flow volume. However, a blood flow volume increase was also observed when joint exercise was performed, which suggests that the blood flow 


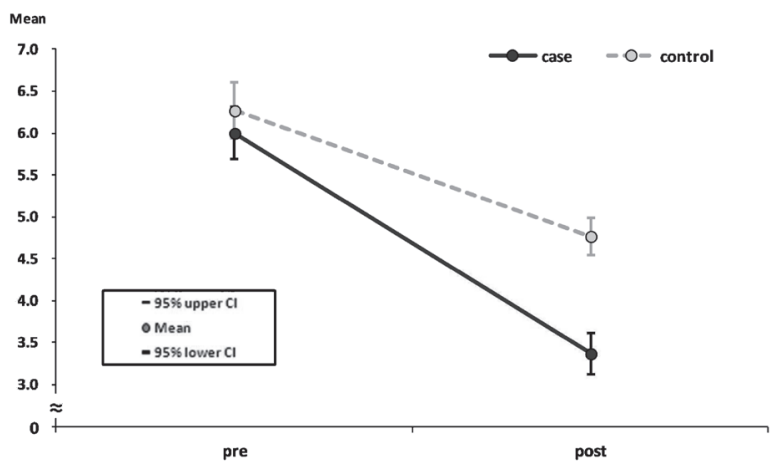

Fig. 1. Average profile of the degree of pain

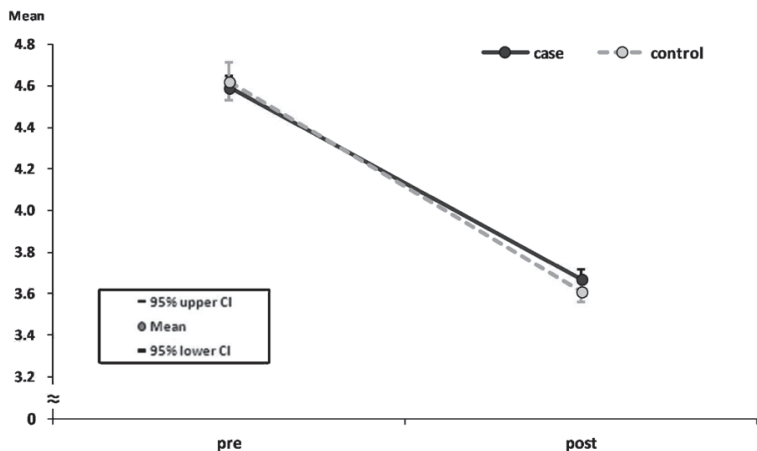

Fig. 2. Average profile of blood flow velocity

Table 1. Multiple comparison of the degree of pain

\begin{tabular}{cccc}
\hline Item & Comparison & $\begin{array}{c}\text { Difference in } \\
\text { Least Square Means }\end{array}$ & 95\% Confidence Interval \\
\hline Experimental group* & Pre-Post & 2.63 & $2.13 \sim 3.13$ \\
Control group* & Pre-Post & 1.50 & $1.00 \sim 2.00$ \\
Pre-test point & $\begin{array}{c}\text { Experimental group- } \\
\text { Control group }\end{array}$ & -0.27 & $-0.77 \sim 0.23$ \\
Post-test point* & Experimental group- & -1.40 & $-1.90 \sim-0.90$ \\
${ }^{*}: \mathrm{p}<0.05$ & Control group & &
\end{tabular}

Table 2. Multiple comparison of blood flow velocity

\begin{tabular}{cccc}
\hline Item & Comparison & $\begin{array}{c}\text { Difference in } \\
\text { Least Square Means }\end{array}$ & 95\% Confidence Interval \\
\hline Experimental group* & Pre-Post & 0.92 & $0.79 \sim 1.05$ \\
Control group* & Pre-Post & 1.00 & $0.88 \sim 1.13$ \\
Pre-test point & $\begin{array}{c}\text { Experimental group-Control } \\
\text { group }\end{array}$ & -0.03 & $-0.16 \sim 0.10$ \\
Post-test point & $\begin{array}{c}\text { Experimental group-Control } \\
\text { group }\end{array}$ & 0.05 & $-0.07 \sim 0.18$ \\
${ }^{*}: \mathrm{p}<0.05$ & & &
\end{tabular}

volume increase is not a treatment effect of Super Lizer. We anticipate that Super Lizer will be beneficial for reduction of pain in rotator cuff patients. Moreover, Super Lizer has one advantage in terms of treatment, which is that no contraindications against it have been reported. Future research should investigate complications in patients with diverse types of muscle injuries. We consider much research will be necessary.

\section{ACKNOWLEDGEMENT}

This research was supported by a Kyungsung University Research Grant in 2012.

\section{REFERENCES}

1) Yoon DM, Oh HG: The use of sticker type temperature indicator in Stellate
Ganglion Block. Korean J Pain, 1994, 7: 49-52.

2) Lee PB, Seo JH, Seong JS, et al.: Can SUPER LIZER alleviate the local pain induced by caudal puncture? J Korean Pain Soc, 2002, 15: 126-131.

3) Bonelli S, Conoscente F, Movilia PG, et al.: Regional intravenous guanethidine vs stellate ganglion block in reflex sympathetic dystrophies a randomizes trial. Pain, 1983, 16: 297-307. [CrossRef]

4) Eiko A, Icho H: SUPER LIZER. Pain Clin, 1998, 19: 49-56.

5) Fukuda H, Hamada K, Nakajima T, et al.: A. Pathology and pathogenesis of the intratendinous tearing of the rotator cuff viewed from en bloc histologic sections. Clin Orthop Relat Res, 1994, 304: 60-67. [Medline]

6) Mitchell C, Adebajo A, Hay E, et al.: Shoulder pain, diagnosis and management in primary care. BMJ, 2005, 331: 1124-1128. [Medline] [CrossRef]

7) Morikawa K, Kawachi A, Matsuo I, et al.: Clinical effect of SUPER LIZER (HA-30). Orie Med Pain Clin, 1992, 22: 37-44.

8) van der Windt DA, Koes BW, de Jong BA, et al.: Shoulder disoeders in general practice: incidence, patient characteristics, and menagement. Ann Rheum Dis, 1995, 54: 959-964. [Medline] [CrossRef]

9) Yoshizawa A: The effect of irradiation of linear polarized light therapy around the area of stellate ganglion for relief of pain. J Bone Jt, 1994, 7: $20-24$. 